\title{
INJURY TO SKELETAL MUSCLES OF MICE BY FORCED LENGTHENING DURING CONTRACTIONS
}

\author{
JOHN A. FAULKNER*, DAVID A. JONES AND JOAN M. ROUND \\ Department of Medicine, University College and Middlesex Hospital Medical School, the Rayne Institute, \\ University Street, London WCIE 6JJ \\ and \\ * Department of Physiology, University of Michigan Medical School, Ann Arbor, Michigan 48109-0622, USA \\ (MANUSCRIPT RECEIVED 24 DECEMBER 1988, ACCEPTED 21 MARCH 1989)
}

\section{SUMMAR Y}

Our purpose was to determine the amount of injury to extensor digitorum longus (EDL) and anterior tibial (ATB) muscles of mice from $1 \mathrm{~h}$ to 30 days following passive shortening and lengthening, shortening contractions and lengthening contractions. The shorteninglengthening cycle was of $600 \mathrm{~ms}$ duration and was repeated every $5 \mathrm{~s}$ for $30 \mathrm{~min}$. Contractions were produced during either the shortening or lengthening phase with stimulation at $100 \mathrm{~Hz}$ for $300 \mathrm{~ms}$. The amount of injury was evaluated by the decrease in the number of fibres in a crosssection of the muscle and in the maximum isometric tetanic force $\left(P_{0}\right)$. Passively shortened and lengthened and actively shortened EDL and ATB muscles showed no evidence of injury. At 3-5 days after lengthening contractions, EDL and ATB muscles showed an inflammatory response. At 3-5 days, the number of fibres in the cross-section of the injured EDL muscles was $65 \%$ of that of the contralateral control EDL muscle, whereas no change was observed in the number of fibres in the injured ATB muscles. By 3 days, the presence of myoblasts, myotubes and central nuclei indicated regeneration of fibres. One hour after shortening contractions, the $P_{0}$ of EDL and ATB muscles decreased to about $75 \%$ of the contralateral control value, but recovered completely by 3 days. In contrast, $1 \mathrm{~h}$ after lengthening contractions the $P_{0}$ of EDL and ATB muscles decreased to 52 and $40 \%$ respectively of the contralateral control value and then both recovered to about $65 \%$ within $3 \mathrm{~h}$. For the ATB muscle, the $P_{0}$ showed a secondary decrease to $49 \%$ of the contralateral control value at 1 day and for the EDL muscle, to $47 \%$ at 3 days. Each muscle recovered gradually and by 30 days the number of fibres and the $P_{0}$ of the injured muscles were not significantly different from values for contralateral control muscles.

\section{INTRODUCTION}

Early observations of injury to skeletal muscle fibres as a result of stretching an active muscle were made by Chauveau (1901) and Hough (1902). Subsequent studies have confirmed that injury is more likely to occur following exercise which involves stretching rather than shortening muscles (Armstrong, Ogilvie \& Schwane, 1983; Newham, McPhail, Mills \& Edwards, 1983a). The injury associated with eccentric exercise has been identified by histological (Armstrong et al. 1983; McCully \& Faulkner, 1985; Jones, Newham, Round \& Tolfree, 1986; Round, Jones \& Cambridge, 1987; Stauber, Fritz, Vogelbach \& Dahlmann, 1988) electron microscopical (Fridén, Sjöstrom \& Ekblom, 1983; Newham et al. 1983a), and physiological (McCully \& Falkner, 1985, criteria. Lengthening exercise is also associated with late-onset muscle soreness widely reported in human subjects (Hough, 1902; Asmussen, 1956; Fridén et al. 1983; Newham, Mills, Quigley \& Edwards, 1983 b).

McCully \& Faulkner (1985) developed an apparatus and experimental protocol that permitted the study of contraction-induced injury of a single muscle under controlled conditions. The procedure involved the cutting and subsequent repair of the distal tendon 
of the extensor digitorum longus (EDL) muscle. The sham-operation of cutting and repairing the tendon resulted in a $25 \%$ decrease in the maximum isometric tetanic force $\left(P_{0}\right)$ at 3 and 5 days, which subsequently recovered to the contralateral control value. The deficit in $P_{0}$ due to the repaired tenotomy complicated the interpretations of the data. Furthermore, the magnitude of the injury immediately following the shortening and lengthening contractions was not investigated. We have developed an apparatus (Faulkner \& Jones, 1985) and a protocol (Faulkner, Jones \& Round, 1985) that permits comparisons of the effect of different forms of exercise on muscle structure and function uncomplicated by tenotomy and repair of the distal tendon. The muscles were exercised by either allowing them to shorten while being stimulated (shortening contractions) or by forcible extension during stimulation (lengthening contractions); these forms of exercise are sometimes referred to as positive and negative work respectively. Our purpose was to follow changes that might occur in the fibre number or the $P_{0}$ of EDL and anterior tibial (ATB) muscles of the mouse during the 30 days following shortening contractions, lengthening contractions and passive lengthening and shortening.

\section{METHODS}

The experiments were performed on 130 mice. Each mouse was used in only one experiment. Each investigator was licensed by the Home Office and the operations and experiments were conducted in accordance with the policy statement on the care and use of laboratory animals published by the American Physiological Society. Mice were anaesthetized by an intraperitoneal injection of an initial dose of $40 \mathrm{mg} / \mathrm{kg}$ sodium pentobarbitone. Supplemental doses were administered as required to maintain an adequate depth of anaesthesia.

The peroneal nerve was exposed and a small hook electrode was inserted under it. The left knee was clamped to the baseplate of the apparatus. The foot was placed in a Perspex holder and taped in place with the ankle joint positioned directly over the pivot (Faulkner \& Jones, 1985).

The shortening and lengthening of the dorsiflexor muscles was accomplished by hooking a lever arm attached to the rim of the driving wheel of a Watson \& Marlow roller pump $(250 \mathrm{~W})$ to the tip of the holder. Rotation of the wheel moved the foot through $100 \mathrm{deg}$ from approximately full extension to almost full flexion and then returned the foot to the starting position. The velocity of the displacement was set so that the complete flexion-extension cycle occurred in $600 \mathrm{~ms}$. The cycle was repeated once every $5 \mathrm{~s}$ for $30 \mathrm{~min}$. For contractions, the EDL and ATB muscles were stimulated at $100 \mathrm{~Hz}$ for $300 \mathrm{~ms}$ with $100 \mu$ s pulses of supramaximum voltage (5-10 V) during either the shortening or the lengthening phase of the anterior tibialis group. For passive shortening and lengthening the stimulator remained switched off throughout the cycle. Sham-operated animals were anaesthetized and the peroneal nerve was exposed but not stimulated. The wound was closed and the animals allowed to recover in the same way as the test animals.

The $P_{0}$ of the dorsiflexors was determined in vivo before and after each experiment. A separate series of experiments was designed to determine the effect of lengthening contractions on the development of force. The six pairs of experimental and contralateral control EDL muscles were removed and force was measured in vitro after each of 10,20 and $30 \mathrm{~min}$ of lengthening contractions. At the end of the exercise, the foot was removed from the holder, the electrodes were disengaged from the peroneal nerve, and the skin was sutured closed. Following closure of the wound, the incision was bathed with betadine and the mouse was then returned to its cage. The mice recovered from the anaesthetic in 15-30 min and resumed normal activities immediately with no evidence of discomfort or locomotor impairment.

After the protocol for contraction-induced injury, the contractile properties of the experimental and the contralateral control, EDL and ATB muscles were measured in vitro at $35^{\circ} \mathrm{C}$ at 1,2 and $3 \mathrm{~h}$ and $1,3,5,7,14$ and 30 days. Mice were anaesthetized as described previously. The dorsiflexor muscles were exposed and lengths of 5-0 nylon suture were tied to the proximal and distal tendons of the EDL muscles and to the distal tendon of the ATB muscles. The ATB muscle has a broad attachment to the tibia. To obtain a secure tie without damaging muscle fibres, the bone was cut and the sutures were tied to the bone. Muscles were removed and placed in a muscle bath filled with 
Table 1. The body wet mass, muscle wet mass, muscle length and maximum isometric tetanic force of contralateral control extensor digitorum longus $(E D L)$ and anterior tibialis $(A T B)$ muscles of female $C D-1$ mice

\begin{tabular}{lccc}
\hline \hline & \multicolumn{2}{c}{ Muscles } \\
\cline { 2 - 3 } & & EDL & ATB \\
\hline Body mass (g) & $25 \cdot 5 \pm 0.6$ & $25 \cdot 5 \pm 0.6$ \\
Muscle mass (mg) & $8 \cdot 0 \pm 1 \cdot 0$ & $47 \cdot 3 \pm 1 \cdot 9$ \\
Muscle length (mm) & $13 \cdot 5 \pm 0 \cdot 7$ & $13 \cdot 1 \pm 0.6$ \\
& Maximum force (mN) & $218 \pm 8$ & $770 \pm 27$ \\
\hline \hline
\end{tabular}

Values are expressed as mean \pm S.E.M.

buffered Krebs bicarbonate solution gassed with $95 \% \mathrm{O}_{2}$ and $5 \% \mathrm{CO}_{2}$. Once the muscles were removed, the anaesthetized mice were killed with a lethal injection of sodium pentobarbitone.

The sutures attached to the tendons of each muscle were tied to a fixed post and to a force transducer. The muscles were stimulated directly by platinum electrodes (Jones, Howell, Roussos \& Edwards, 1982). The length of the muscle was adjusted until maximal twitch force was developed $\left(L_{0}\right)$. Previous experiments have shown that maximum tetanic force is also achieved at $L_{0}$ (Brooks \& Faulkner, 1988). All subsequent experiments were carried out at $L_{0}$. A frequency-force relationship was determined for each muscle by stimulating at $10,30,50,80,100,150,200 \mathrm{~Hz}$, or higher if necessary, to obtain a plateau of force. The maximum force developed was defined as $P_{0}$. Five or six pairs of experimental and contralateral control muscles were studied at each time period.

Following the measurement of contractile properties, some experimental and contralateral control muscles were selected for histological examination. A cross-sectional segment cut from the belly of the muscle was oriented vertically on a cork disc, supported in mounting medium, and frozen in isopentane cooled with liquid nitrogen. Cryostat sections were cut at $8 \mu \mathrm{m}$ and stained with haematoxylin and eosin. The total muscle cross-sectional area of the EDL and ATB muscles was determined by planimetry. For both the EDL and ATB muscles at each time period, a Lietz image analyser was used to measure mean fibre cross-sectional areas of samples of identifiable fibres. The image analyser was also used to obtain counts of the number of intact fibres in a cross-section of experimental and contralateral control EDL muscles. Since there was no evidence of any change in the fibre number in the ATB muscles at any time period after the lengthening contractions the number of fibres were counted in ten muscles selected at random. For the EDL muscles, the ratio of the area of uninjured fibres (number of fibres $\times$ mean fibre area) to the total area of the muscle was estimated.

Means and standard errors of the mean were determined for all data sets and statistical differences were determined by Student's $t$ test. Significance was set $a$ priori at a probability level of 0.05 .

\section{RESULTS}

The control values for body masses of the mice, muscle wet mass, muscle length, and $P_{0}$ of the EDL and ATB muscles are presented in Table 1. One hour after the passive shortening and lengthening protocol the EDL and ATB muscles showed no histological evidence of injury to skeletal muscle fibres (Fig. $1 A$ and $D$ ) and the $P_{0}$ of the passively shortened and lengthened muscles was not significantly different from that of the contralateral control muscles (Table 2). The lack of a difference between the passively shortened and lengthened muscles and the contralateral control muscles indicated that neither the operative procedures nor passive shortening and lengthening caused fatigue or injury to skeletal muscle fibres. Therefore, the contralateral control muscles provided satisfactory control data for the subsequent experiments on shortening and lengthening contractions and the 

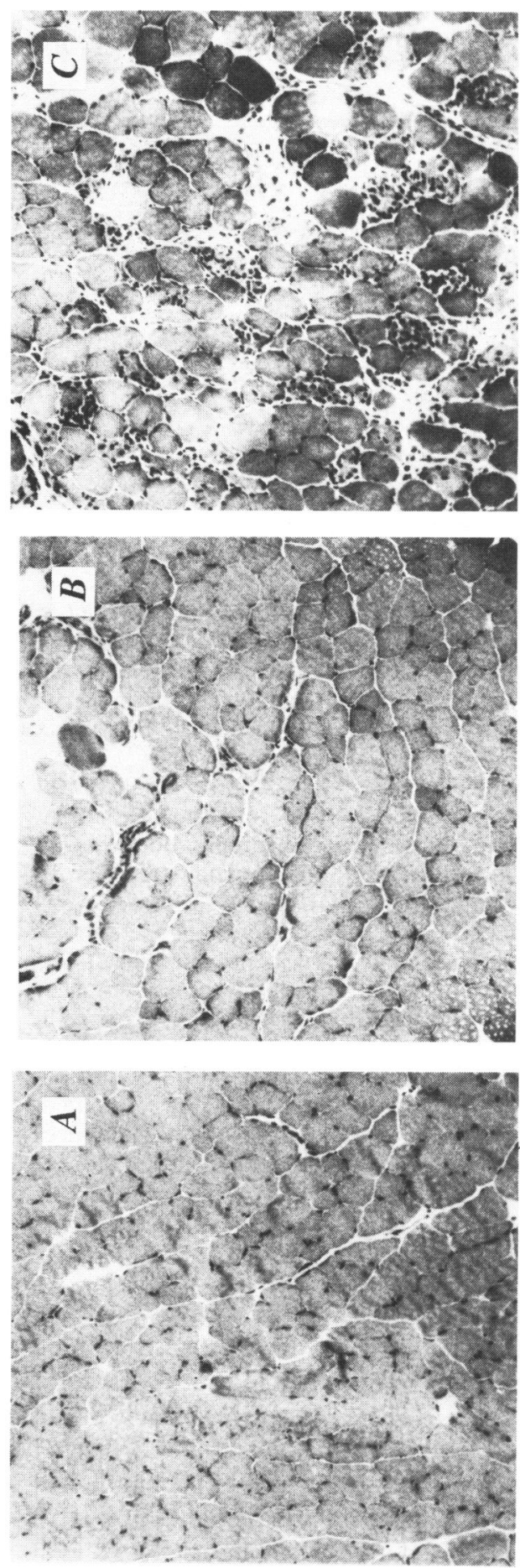
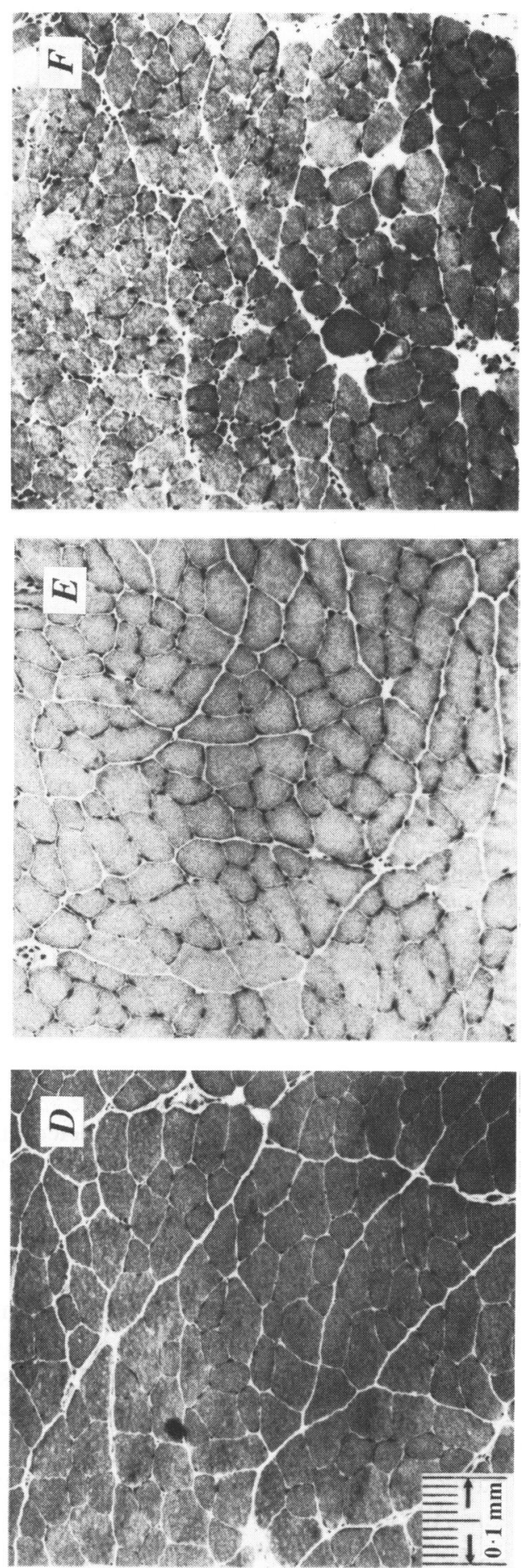

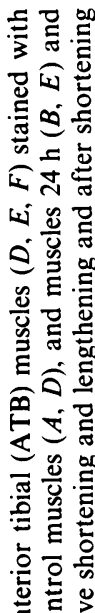
हี ठ․ 푼

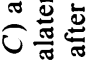
si उ广 s. 总它 हु กับ 包造 品 늠

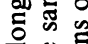
छ 흔

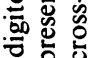
完总 खै 4 象 政

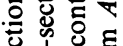
出它 它 원 $\leftarrow$ F 등 象运要 윤

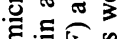
읏․ㅇ

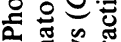
- 结要吉 的实 
Table 2. The maximum isometric tetanic force of EDL and ATB muscles $1 \mathrm{~h}$ after $30 \mathrm{~min}$ of passive shortening and lengthening and $1 \mathrm{~h}$ after and 3 days after 30 min of shortening contractions or 30 min of lengthening contractions.

\begin{tabular}{llllll}
\hline \hline & & & & 3 days \\
\cline { 3 - 6 } & EDL & ATB & & EDL & ATB \\
\hline Muscles passively shortened and lengthened (5) & $94 \pm 7$ & $.98 \pm 5$ & & - & - \\
Muscles shortened during contraction (6) & $76 \pm 6^{*}$ & $73 \pm 7^{*}$ & & $96 \pm 4$ & $91 \pm 5$ \\
Muscles lengthened during contraction (6) & $57 \pm 8^{*}$ & $40 \pm 5^{*}$ & & $47 \pm 5^{*}$ & $63 \pm 5^{*}$ \\
\hline
\end{tabular}

Values are expressed as a percentage of the value for the contralateral control muscle, mean \pm S.E.M.; * significantly different from the values for contralateral control muscles $(P<0.05)$. Number of muscles is shown in parentheses.

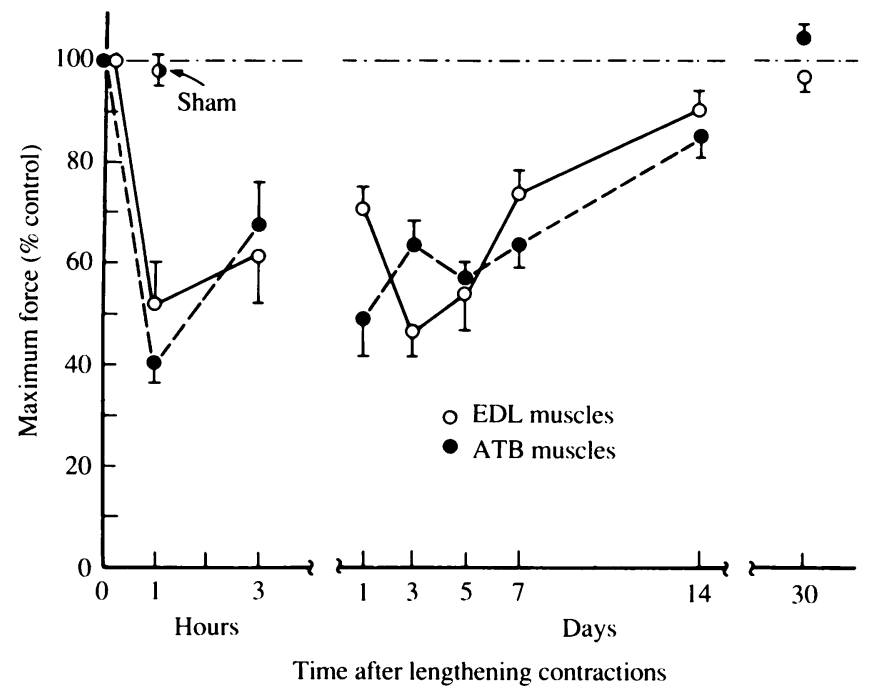

Fig. 2. The maximum isometric tetanic force of extensor digitorum longus (EDL) and anterior tibial (ATB) muscles at selected times after lengthening contractions expressed as a percentage of the contralateral control muscles. Values for the EDL and ATB muscles after passive shortening and lengthening and after shortening contractions were not different from those for contralateral control muscles. Values for sham-operated animals shown at $1 \mathrm{~h}$ and 30 days.

$P_{0}$ of experimental muscles were expressed as a percentage of the contralateral control muscle (Fig. 2).

No histological evidence of injury was observed for contralateral control EDL and ATB muscles at any time period (Fig. $1 A$ and $D$ ). Similarly the EDL and ATB muscles, that underwent passive shortening or lengthening movements presented no histological evidence of injury at any time nor was there any evidence of damage in muscles that had actively shortened (data not shown). There was no evidence of damage in either type of muscle $24 \mathrm{~h}$ following active lengthening contractions (Fig. $1 B$ and $E$ ). Compared to the $P_{0}$ of the contralateral control muscles $1 \mathrm{~h}$ after the protocol, the $P_{0}$ of muscles that were passively shortened and lengthened were not significantly different, whereas the $P_{0}$ of EDL and ATB muscles decreased significantly after shortening contractions (Table 2). After shortening contractions the $P_{0}$ returned to contralateral control values by 3 days. 
Table 3. The total cross-sectional area through the belly of the muscle at right angles to the long axis of the muscle, the ratio of the area of uninjured fibres (mean fibre area $\times$ number of fibres) to the total cross-sectional area through the belly of the muscle, the number of fibres in the total muscle cross-section, and the mean cross-sectional area of individual fibres in this cross-section. Data are for the contralateral control extensor digitorum longus $(E D L)$ and anterior tibial $(A T B)$ muscles

\begin{tabular}{lcccc}
\hline & Control & $1 \mathrm{~h}$ & $24 \mathrm{~h}$ & $3-5$ days \\
\hline EDL muscles & $(7)$ & $(4)$ & $(4)$ & $(6)$ \\
& & & & \\
Muscle area $\left(\mathrm{mm}^{2}\right)$ & $1 \cdot 84 \pm 0 \cdot 1$ & $1 \cdot 69 \pm 0 \cdot 07$ & $1 \cdot 87 \pm 0 \cdot 07$ & $1 \cdot 12 \pm 0 \cdot 2^{*}$ \\
Ratio of area $(\%)$ & $94 \pm 3$ & $96 \pm 4$ & $94 \pm 3$ & $72 \pm 6^{*}$ \\
Fibre area $\left(\mu \mathrm{m}^{2}\right)$ & $1390 \pm 60$ & $1280 \pm 40$ & $1550 \pm 85$ & $1750 \pm 180^{*}$ \\
Number of fibres & $1300 \pm 70$ & $1180 \pm 105$ & $1150 \pm 105$ & $650 \pm 60^{*}$ \\
ATB muscles & $(11)$ & $(4)$ & $(4)$ & $(7)$ \\
& & & & \\
Muscle area $\left(\mathrm{mm}^{2}\right)$ & $5 \cdot 63 \pm 0 \cdot 24$ & $5 \cdot 92 \pm 0 \cdot 18$ & $6 \cdot 26 \pm 0.30$ & $5 \cdot 37 \pm 0 \cdot 33$ \\
Fibre area $\left(\mu \mathrm{m}^{2}\right)$ & $1550 \pm 55$ & $1820 \pm 95$ & $2005 \pm 125^{*}$ & $1550 \pm 40$ \\
Number of fibres & $2950 \pm 100$ & - & - & - \\
\hline \hline
\end{tabular}

Values are mean \pm S.E.M.; * significantly different from the contralateral control value $(P<0 \cdot 05)$. Number of muscles is shown in parentheses. Note: no significant differences were observed in data collected at 3 and 5 days and data on these two days were pooled. Similarly, no difference occurred in the number of fibres in the crosssection of the ATB muscle and twelve muscles were chosen randomly for representative fibre counts.

After lengthening of active muscles the cross-sections obtained from EDL and ATB muscles at 3-5 days showed evidence of an inflammatory response with infiltration of the muscle by a large population of mononuclear cells (Fig. $1 C$ and $F$ ). The EDL muscles showed a significantly greater amount of injury than the ATB muscles. No significant differences were observed between results at 3 and 5 days for EDL or ATB muscles in muscle cross-sectional area, ratio of fibre area to total muscle area (EDL muscle only), mean fibre area, and number of fibres. Consequently, the data for these two days were pooled for each muscle. Between 3 and 5 days after the exercise, the area occupied by what appeared to be uninjured skeletal muscle fibres constituted $70 \%$ of the total cross-sectional area of the EDL muscle and the number of fibres was $50 \%$ of the value for contralateral control EDL muscles (Table 3). Although the ATB muscles showed an inflammatory response at this time, no loss of fibres or decrease in the overall area occupied by fibres was observed. By 30 days after the lengthening contractions, no evidence of injury was seen in the histological sections of either the EDL or the ATB muscles.

The mean area for fibres in contralateral control EDL and ATB muscles is in reasonable agreement with previously published values on mouse limb muscles (Timson, Bowlin, Dudenhoeffer \& George, 1985). A significant increase in the mean fibre area of $25-30 \%$ occurred in the EDL muscle at 3 days and in the ATB muscle at $24 \mathrm{~h}$ (Table 3). The increased area appeared to result from the presence of large swollen fibres (Fig. $1 C$ and $E$ ). The ratio of the area composed of fibres to the total cross-sectional area of the EDL muscle decreased significantly at 3 days. This analysis was not performed for the ATB muscle since no loss of fibres occurred.

During the $30 \mathrm{~min}$ of lengthening contractions, the force developed by the dorsiflexor muscles declined significantly to $58 \%$ of the initial force by $10 \mathrm{~min}, 51 \%$ by $20 \mathrm{~min}$, and 

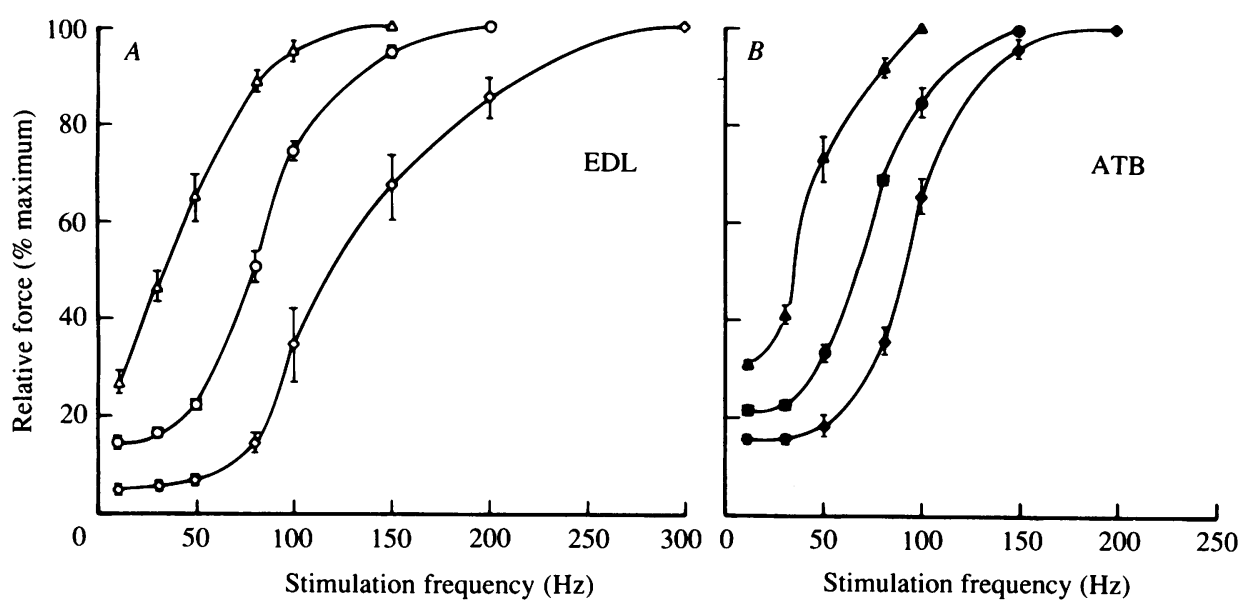

Fig. 3. The frequency-force curves for extensor digitorum longus (EDL, $A$, open symbols) and anterior tibial (ATB, $B$, filled symbols) for contralateral control muscles and for exercised muscles $1 \mathrm{~h}$ and 5 days after the lengthening contractions. $\bigcirc,-$ control muscle; $\diamond, \diamond$ muscle $1 \mathrm{~h}$ after lengthening contractions; $\triangle, \boldsymbol{\Delta}$ muscle 5 days after lengthening contractions.

$40 \%$ by $30 \mathrm{~min}$. From $1 \mathrm{~h}$ to 14 days after muscles underwent lengthening contractions, the values for $P_{0}$ for both the EDL and ATB muscles were decreased significantly compared to the values for the contralateral control muscle (Fig. 2). The lowest value for $P_{0}$ was recorded at 1 day for the ATB muscle and at 3 days for the EDL muscle. Following the nadir, the $P_{0}$ of both muscles increased gradually until, by 30 days, the values for $P_{0}$ were not significantly different from the values for the contralateral control muscles.

The frequency-force relationship of the contralateral control muscles showed a characteristic sigmoid shape with the plateau at $200 \mathrm{~Hz}$ for the EDL muscle and at $150 \mathrm{~Hz}$ for the ATB muscle (Fig. 3). The frequency-force relationship for muscles $1 \mathrm{~h}$ after active lengthening contractions was shifted downwards and to the right and $P_{0}$ occurred at $300 \mathrm{~Hz}$ for the EDL muscle and at $200 \mathrm{~Hz}$ for the ATB muscle. Five days after active lengthening contractions, the frequency-force relationship was shifted upwards and to the left with the $P_{0}$ being obtained at $150 \mathrm{~Hz}$ for the EDL muscle and at $100 \mathrm{~Hz}$ for the ATB muscle.

\section{DISCUSSION}

In a previous study of contraction-induced injury to the EDL muscles of mice (McCully \& Faulkner, 1985), tenotomy and repair of the distal tendon of passively shortened and lengthened muscles resulted in a $25 \%$ decrease in the $P_{0}$ compared to the contralateral control muscles. In the present study, the lack of any significant difference in histological appearance or in $P_{0}$ between passively shortened and lengthened muscles and contralateral control muscles permitted the use of the contralateral muscles as the controls and facilitated the interpretation of the structural and functional indices of injury. The similarity in the shape of the time-dependent curves for the deficit in $P_{0}$ following lengthening contractions and in the magnitude of the deficit support the premise of McCully \& Faulkner (1985) that the sham operation of tenotomy and repair provided a satisfactory control muscle. 
The decrease of about $25 \%$ in the $P_{0}$ of EDL and ATB muscles $1 \mathrm{~h}$ after shortening contractions with no histological evidence of injury to muscle fibres suggests that the decrease in $P_{0}$ (which recovers within 3 days) following shortening contractions is qualitatively different to the deficit caused by lengthening contractions. Three days after lengthening contractions the histological criteria of injury and the deficit in $P_{0}$ were most severe. The lack of microscopic evidence of injury following shortening contractions is consistent with observations of shortening contractions by single muscles of mice (McCully \& Faulkner, 1985) and of concentric exercise performed by rats (Vihko, Salminen \& Rantamaki, 1979; Armstrong et al. 1983), and humans (Fridén et al. 1983; Newham et al. $1983 b$; Newham, Jones \& Edwards, 1986).

Both - muscles showed evidence of a biphasic change in $P_{0}$ following exercise and subsequent recovery (Fig. 2). The significance of these fluctuations in force is not clear but they could indicate the presence of two processes. One form may be fairly rapidly reversible, does not entail structural damage and is possibly analogous to the loss of force seen following shortening contractions. The second has a longer time course and involves physical damage to muscle fibres.

The cause of injury to skeletal muscle fibres during lengthening contractions is not known. The high correlation between the peak force during lengthening contractions and the magnitude of the injury (McCully \& Faulkner, 1986) supports the premise (Hough, 1902; Newham et al. $1983 a$; McCully \& Faulkner, 1985) that high forces, often $150 \%$ or more than $P_{0}$, result in a mechanical disruption of ultrastructural elements of skeletal muscle fibres although recent evidence suggests that muscles working at long length may be at risk (Newham, Jones, Ghosh \& Aurora, 1988). Armstrong et al. (1983) have postulated that the injury occurs to a population of stress-susceptible fibres in a muscle, but the specific characteristics of fibres that might make them more susceptible to contractioninduced injury have not been identified. The mechanism responsible for the delayed-onset injury might be an influx of calcium (Jackson, Jones \& Edwards, 1984; Jones, Jackson, McPhail \& Edwards, 1984), or oxygen-free radical damage (Jackson, Jones \& Edwards, 1985).

The loss of $34 \%$ of the fibres from the histological sections of EDL muscles at 3 days after lengthening contractions, where there was no evidence of fibre loss in sections of ATB muscles at any time, could be a consequence of the differences in fibre lengths. The length of fibres in the EDL muscle of the mouse is about $6 \mathrm{~mm}$ (Brooks \& Faulkner, 1988). Measurements of twenty-five fibres from each of two contralateral control ATB muscles following nitric acid digestion provided a fibre length-muscle length ratio of 0.71 and an estimated fibre length of $9.3 \mathrm{~mm}$. The dorsiflexor muscles were lengthened about $1.5 \mathrm{~mm}$ during the lengthening contractions. Consequently, the fibres of the EDL muscle were lengthened about $28 \%$ of their length, whereas the longer fibres in the ATB muscle were only lengthened $16 \%$. The velocity of the stretch, in terms of muscle lengths per second, would also differ in a similar way for the two muscles. Another factor that might contribute to the difference between two muscles is their fibre type composition. ATB muscles contain more slow fibres than the EDL muscles and although we have no evidence from the present results that one fibre type was more affected, there is a suggestion from human work that fast fibres may be more susceptible to this form of damage (Jones et al. 1986).

The ATB muscle showed a $51 \%$ loss in $P_{0}$ at 1 day with no loss in fibre number, whereas the EDL muscle showed a $53 \%$ decrease in $P_{0}$ at 3 days with a $35 \%$ loss of fibres. In each case, the deficit in the development of force was significantly greater than the evidence of overt injury to fibres by histological evaluation. Many fibres that appear normal in one 
histological section show signs of injury in another section. In addition, Fridén et al. (1983) have shown that significantly more fibres are classified as injured by electron microscopy than by light microscopy. Since a quantitative assessment of fibre injury by electron microscopy is not possible, the indirect measurement of the degree of functional impairment by the deficit in $P_{0}$ appears to offer the most promising evaluation. The $P_{0}$ has been used effectively to assess the functional deficit following free whole muscle transplantation and the recovery as skeletal muscle fibres regenerate (Carlson \& Faulkner, 1983).

Our observation of decreased relative forces at low frequencies of stimulation $1 \mathrm{~h}$ after lengthening contractions are consistent with reports of a shift to the right of the frequency-force relationship after lengthening contractions of muscles in frogs (Katz, 1939), mice (McCully \& Faulkner, 1985) and human beings (Newham et al. 1983 b). This phase was followed by a shift to the left, giving increased relative forces at low frequencies of stimulation, as observed 5 days after lengthening contractions. A similar shift to the left is seen when fibres are regenerating following free whole muscle grafting (Faulkner, Niemeyer, Maxwell \& White, 1980). Regenerating fibres invariably have prolonged contraction and half-relaxation times (Carlson \& Faulkner, 1983). Whether the upward shift in the frequency-force relationship following contraction-induced injury results from selective injury to fast fibres (Jones et al. 1986), the synthesis of slower isoforms of myosin by regenerating fibres (Hoh, McGrath \& White, 1976), or both has not been determined.

Regeneration of skeletal muscle fibres has been reported following a variety of different types of injury (Carlson \& Faulkner, 1983). A significant amount of regeneration must have occurred in the injured EDL muscle since between 3 and 5 days the number of fibres in the belly of the muscle decreased by $45 \%$ (McCully \& Faulkner, 1985). The disappearance of fibres could result from either focal or total degeneration of fibres.

The lack of any fibre loss in the belly of the ATB muscles might reflect focal injury to other parts of the muscle or injury not observed at the level of light microscopy (Fridén et al. 1983). An injury clearly not evident at the light microscope level is present in both muscles during the first $24 \mathrm{~h}$. The appearance of myoblasts and myotubes in histological sections of EDL muscles within 3-5 days of the contraction-induced injury and the restoration of contralateral control values for fibre number by 30 days suggests that regeneration of fibres constitutes the major repair process following contraction-induced injury. Whether repair of the more subtle injury to fibres in the ATB muscles involves regeneration cannot be determined from the measures used in this study.

James M. Kneebone assisted in the quantitative analysis of the histological sections. The research was supported by a grant from the National Institute on Ageing AG-06157 (J.A.F.) and with support from the Muscular Dystrophy Group of Great Britain (D.A.J.).

\section{REFERENCES}

Armstrong, R. B., Ogilvie, R. W. \& Schwane, J. A. (1983). Eccentric exercise-induced injury to rat skeletal muscle. Journal of Applied Physiology 54, 80-93.

ASMUSSEN, E. (1956). Observations on experimental muscular soreness. Acta rheumatologica scandinavica 2, 109-116.

Brooks, S. V. \& FAULKNER, J. A. (1988). Contractile properties of skeletal muscles from young, adult and aged mice. Journal of Physiology 404, 71-82.

CARlson, B. M. \& FAULKNER, J. A. (1983). The regeneration of skeletal muscle fibres following injury: a review. Medicine and Science in Sports and Exercise 15, 187-198. 
Chauveau, S. (1901). La dépense énergétique de l'entraînement respective le travail motor et le travail résistant de l'homme qui s'élève au descent sur la roue de Hirn. Comptes rendus hebdomadaire des séances de l'Académie des sciences 132, 194-202.

FAulKner, J. A. \& Jones, D. A. (1985). An apparatus for unilateral in situ exercise of the lower limb muscles of small muscles. Journal of Physiology 365, $11 P$.

FaulKner, J. A., Jones, D. A. \& Round, J. (1985). Injury to skeletal muscle of mice by lengthening contractions. Journal of Physiology 365, $75 P$.

Faulkner, J. A., Niemeyer, J. A., Maxwell, L. C. \& White, T. P. (1980). Contractile properties of transplanted extensor digitorum longus muscles of cats. American Journal of Physiology 238 (Cell Physiology 7), C120-126.

Fridén, J., Suöstrom, M. \& Ekblom, B. (1983). Myofibrillar damage following intense eccentric exercise in man. International Journal of Sports Medicine 4, 170-176.

HoH, J. F. Y., MCGRATH, P. A. \& WHITE, R. I. (1976). Electrophoretic analysis of multiple forms of myosin in fast-twitch and slow-twitch muscles of the chick. Biochemistry Journal 157, 87-95.

Hough, T. (1902). Ergographic studies in muscular soreness. American Journal of Physiology 7, 76-92.

JACKSON, M. J., JONES, D. A. \& EdWARDS, R. H. T. (1984). Experimental skeletal muscle damage: the nature of the calcium-activated degenerative process. European Journal of Clinical Investigation 14, 369-374.

JACKSON, M. J., Jones, D. A. \& Edwards, R. H. T. (1985). Vitamin E and muscle disease. Journal of Inherited Metabolic Diseases 8, suppl. 1, 84-87.

Jones, D. A., Howell, S., Roussos, C. \& Edwards, R. H. T. (1982). Low frequency fatigue in isolated skeletal muscles and the effects of methylxanthines. Clinical Science 63, 161-167.

Jones, D. A., Jackson, M. J., McPhail, G. \& Edwards, R. H. T. (1984). Experimental mouse muscle damage: the importance of external calcium. Clinical Science 66, 317-322.

Jones, D. A., Newham, D. J., Round, J. M. \& Tolfree, S. E. J. (1986). Experimental human muscle damage: morphological changes in relation to other indices of damage. Journal of Physiology 375, 435-448.

KATZ, B. (1939). The relation between force and speed in muscular contraction. Journal of Physiology 96, 45-64.

LuFF, A. R. (1981). Dynamic properties of the inferior rectus, extensor digitorum longus, diaphragm and soleus muscles of the mouse. Journal of Physiology 313, 161-171.

McCully, K. K. \& Faulkner, J. A. (1985). Injury to skeletal muscle fibres of mice following lengthening contractions. Journal of Applied Physiology 59, 119-126.

MCCully, K. K. \& Faulkner, J. A. (1986). Characteristics of lengthening contractions associated with injury to skeletal muscle fibres. Journal of Applied Physiology 61, 293-299.

Newham, D. J., Jones, D. A. \& Edwards, R. H. T. (1986). Plasma creatine kinase changes after eccentric and concentric contractions. Muscle and Nerve 9, 59-63.

Newham, D. J., Jones, D. A., Ghosh, G. \& Aurora, P. (1988). Muscle fatigue and pain after eccentric contractions at long and short length. Clinical Science 74, 553-557.

Newham, D. J., McPhail, G., Mills, K. R. \& Edwards, R. H. T. (1983a). Ultrastructural changes after concentric and eccentric contractions of human muscle. Journal of the Neurological Sciences 61, 109-122.

Newham, D. J., Mills, K. R., Quigley, B. M. \& Edwards, R. H. T. (1983b). Pain and fatigue after concentric and eccentric contractions. Clinical Science 64, 55-62.

Round, J. M., Jones, D. A. \& CAMBridge, G. (1987). Cellular infiltrates in human skeletal muscle: exercise-induced damage as a model for inflammatory muscle disease? Journal of the Neurological Sciences 82, 1-11.

Stauber, W. J., Fritz, V. K., Vogelbach, D. W. \& Dahlmann, B. (1988). Characterisation of muscles injured by forces lengthening: 1. Cellular infiltrates. Medicine and Science in Sports and Exercise 20, 345-353.

Timson, B. F., Bowlin, B. K., Dudenhoeffer, G. A. \& George, J. B. (1985). Fibre number, area, and composition of mouse soleus muscle following enlargement. Journal of Applied Physiology 58, 619-624.

Vihko, V., Salminen, A. \& Rantamaki, A. (1979). Exhaustive exercise, endurance training and acid hydrolase activity in skeletal muscle. Journal of Applied Physiology. 47, 43-50. 